\title{
Évaluer les priorités et les ressources : un exercice d'équilibre
}

\author{
par Tania Mysak
}

$S_{\text {oi }}^{e}$ serrer la ceinture. Travailler plus intelligemment, pas plus fort. Vivre selon ses moyens. Il y a de bonnes chances que vous ayez déjà entendu ces phrases ou des versions de celles-ci. Qu'il s'agisse des budgets fédéraux, des finances familiales ou de l'efficacité en milieu de travail, l'on s'attend à ce que nous réévaluions nos pratiques actuelles et que nous cherchions des façons de les rendre plus viables.

Ce processus est très semblable aux choix d'interactions que les pharmaciens font tous les jours dans leur prestation de soins aux patients. Nous établissons un ordre de priorité et planifions les heures limitées dont nous disposons chaque jour pour être aussi efficaces que possible et pour faire en sorte que ceux qui ont le plus besoin de nos soins les reçoivent. Nous fouillons les ressources disponibles aux patients pour nous assurer qu'ils reçoivent la pharmacothérapie la plus avantageuse qu'ils puissent se permettre. Une gestion adéquate des médicaments permet de s'assurer que les médicaments ajoutés à la pharmacothérapie ont une valeur réelle pour les patients et leurs objectifs de santé. S’il y a un médicament inutile dans la pharmacothérapie, il faut déprescrire : garder l'utile, éliminer le gaspillage.

Du point de vue de la gestion de pharmacie, nous appliquons également ces principes. Les gestionnaires et les directeurs de pharmacie doivent prendre en compte, pour ce qui touche à leur service, quelles unités recevront des soins proactifs, notamment par l'intégration de pharmaciens dans les équipes de soins, et quelles unités auront droit à une approche plus réactive, peut-être à partir de la pharmacie ou à distance. Ils doivent déterminer le niveau d'allocation de service qui peut être offert compte tenu des ressources disponibles et ils doivent établir comment prioriser les exigences toujours changeantes concernant la distribution de médicaments sûrs.

Ultimement ces principes servent à prendre des décisions judicieuses pour ce qui est de répartir des ressources limitées.

À titre d'association professionnelle ayant comme mandat de servir ses membres, la Société canadienne des pharmaciens d'hôpitaux (SCPH) n'est pas à l'abri des exigences de révision des programmes compte tenu de ses ressources limitées. Nos principales sources de revenus en tant que société proviennent des cotisations et de la Conférence sur la pratique professionnelle, qui est lucrative grâce au généreux soutien de l'industrie. Nous faisons toujours face à des défis sur ces deux fronts et travaillons activement de concert avec nos sections et nos partenaires de l'industrie pour maintenir et augmenter ces sources de revenus. Tout en cherchant des occasions dans ces sphères, nous devons aussi tenir compte de notre programmation et nous occuper des passifs du bilan. Cet été, le conseil de la SCPH a commencé à évaluer nos nombreux programmes, les soumettant à une série de questions standards conçues pour juger la valeur que chaque programme offre à la Société. À mesure que cette évaluation avance, nous vous consulterons, vous les membres, pour connaître votre opinion. Ce sont des conversations délicates. Nous savons que, comme dans les exemples fournis ci-dessus, il est très difficile de dire « non " à quelque chose que l'on perçoit personnellement comme important ou que l'on fait depuis longtemps.

Le conseil de la SCPH adhère à la vision de la Société, soit d'être une "société dynamique, en constante évolution ", et à notre objectif stratégique d'équilibre entre les priorités et les ressources. Nous nous ferons un plaisir de vous informer de nos progrès tout au long de cet important travail.

[Traduction par l'éditeur]

Tania Mysak, BSP, Pharm. D., est devenue présidente désignée et agente de liaison de la Société canadienne des pharmaciens d'hôpitaux lors de la réunion du conseil qui a suivi son élection à l'Assemblée générale annuelle en octobre 2018. 